\title{
Nonlinear mechanical response of supercooled melts under applied forces
}

\author{
Heliana Cárdenas ${ }^{1}$, Fabian Frahsa ${ }^{2}$, Sebastian Fritschi ${ }^{2}$, Alexandre Nicolas ${ }^{3}$, \\ Simon Papenkort ${ }^{1}$, Thomas Voigtmann ${ }^{1,4, a}$, and Matthias Fuchs ${ }^{2, \mathrm{~b}}$ \\ 1 Institut für Materialphysik im Weltraum, Deutsches Zentrum für Luft- und Raumfahrt \\ (DLR), 51170 Köln, Germany \\ 2 Fachbereich Physik, Universität Konstanz, 78457 Konstanz, Germany \\ 3 LPTMS, CNRS \& Université Paris-Sud, 91405 Orsay, France \\ ${ }^{4}$ Institut für Theoretische Physik, Heinrich-Heine-Universität Düsseldorf, \\ 40204 Düsseldorf, Germany
}

Received 24 April 2017 / Received in final form 13 June 2017 Published online 10 August 2017

\begin{abstract}
We review recent progress on a microscopic theoretical approach to describe the nonlinear response of glass-forming colloidal dispersions under strong external forcing leading to homogeneous and inhomogeneous flow. Using mode-coupling theory (MCT), constitutive equations for the rheology of viscoelastic shear-thinning fluids are obtained. These are, in suitably simplified form, employed in continuum fluid dynamics, solved by a hybrid-Lattice Boltzmann (LB) algorithm that was developed to deal with long-lasting memory effects. The combined microscopic theoretical and mesoscopic numerical approach captures a number of phenomena far from equilibrium, including the yielding of metastable states, process-dependent mechanical properties, and inhomogeneous pressure-driven channel flow.
\end{abstract}

\section{Introduction}

Precisely 150 years ago, Maxwell laid the foundations for the understanding of viscoelasticity in quiescent liquids [1]. A viscoelastic liquid exhibits a slow structural process characterized by the (final) relaxation time $\tau$ and behaves like a solid with (shear) elastic modulus $G_{\infty}$ for rapid deformations, which correspond to frequencies $\omega$ larger than the inverse relaxation time, viz. $\omega \tau \gg 1$. A glass state is probed when $\tau$ exceeds the experimental time window. It took more than a century until 1984, that the first microscopic theory was presented which derives Maxwell's phenomenology starting from the Newtonian dynamics of the liquid's molecules $[2,3]$. The mode-coupling theory of the glass transition (MCT) identifies the hindering of particles in neighbor-cages as the dominant feedback mechanism that slows down the motion upon lowering the temperature or increasing the density [4]. In (low) molecular liquids, activated dynamics, which is neglected in the (idealized) MCT, enables

\footnotetext{
a e-mail: thomas.voigtmann@dlr.de

b e-mail: matthias.fuchs@ukn.de
} 
particles to escape their cages close to the MCT glass transition, which causes the final relaxation to differ from MCT predictions. In dense colloidal dispersions and possibly other soft amorphous materials, however, activated processes presumably are negligible compared to motion induced by external mechanical fields. Thus the competition between the internal structural dynamics and external driving can be studied in detail [5].

A description of macroscopic flow behavior usually employs phenomenological material laws that provide a closure relation for the Navier-Stokes equations by relating the material's stress tensor to the flow fields. The Maxwell model for the macroscopic stress response close to equilibrium has been the basis for phenomenological extensions to flowing complex fluids [6]. The postulated "constitutive equations" satisfy conditions dictated by symmetry, conservation laws and other general concepts, yet are arbitrary otherwise. Deriving constitutive equations for the nonlinear mechanical response of glass-forming colloidal dispersions is the aim of the generalization of MCT to driven states. It starts from nonlinear-response theory and applies decoupling approximations to force-correlation functions, which are familiar from the theory of slow dynamics in fluids since the work by Kawasaki (e.g., [7]). In its most general form valid for time-dependent but homogeneous flow-driven colloidal states, the approach was worked out in reference [8]. The central assumption, valid close to the glass transition, is that structural relaxation of density fluctuations dominates the slow dynamics. The MCT approach to nonlinear response theory is not restricted to colloidal suspensions: related developments deal with thermostatted non-colloidal systems $[9,10]$.

The mode-coupling theory is to date the only fully microscopic approach to describe the nonlinear response of mechanically driven glass-forming liquids; other approaches, in particular for the deformation of amorphous solids, start from a more mesoscopic point of view [11]. Generalizations of density functional theory to driven states $[12,13]$ capture rapid spatial variations, but miss the slow structural relaxation crucial to glassy arrest [14] or require the development of novel closures [15]. Generalizations of MCT to capture inhomogeneities ("iMCT") have led to scaling-laws for the leading nonlinearities in various response functions, thought to be connected to the size of dynamically correlated regions in supercooled liquids [16,17]. Still, detailed quantitative results are lacking. (We will compare the scaling laws under oscillatory shear with those from iMCT in Sect. 2.4.) Predictions from replica theory exist on linear response quantities like the shear modulus $[18,19]$, but the different closure approximations that are required do not yet provide a consistent picture. The limit of infinite dimensions has recently been discussed as a simplified venue to understand glassy behavior, and first results for sheared states have been derived [20]. Yet, this approach applies to states deep in the glass domain far removed from the viscoelastic regime of interest here.

In this mini-review, we will summarize recent progress regarding the generalization of MCT to the nonlinear response of force-driven and inhomogeneous flows. On the microscopic scale, the extension of the theory to include spatial inhomogeneity and the role of spatial correlations within the theory are discussed. On the macroscopic scale, the development of numerical tools to couple spatially and temporally non-local constitutive equations (such as those arising from MCT) to a continuum-mechanics description is discussed. Pressure-driven flow through a rough channel provides a simple test case where the microscopic theory and MCT-based equations can be compared to particle-based simulations and macroscopic fluid dynamics.

The paper is organized as follows: Section 2 summarizes MCT including its recent extension to inhomogeneous flow. The force-driven yielding due to a macroscopic applied stress and due to a localized force will be discussed in Sections 2.1 and 2.2. The latter case reveals the emergence of a growing length when approaching 
the threshold force for yielding. The solidity length scale over which solid-like longranged elastic stress and strain correlations persist in the fluid, will be discussed in Section 2.3. In Section 2.4, we discuss the break-down of linear response and the leading nonlinear response at the third harmonic under oscillatory driving, contrasting our results to the iMCT scaling laws. Section 3 summarizes our approach to a combined microscopic-theory and macroscopic-fluid-dynamics description of the flow of glass-forming systems. The extension of the Lattice Boltzmann (LB) method to solve the low-Mach-number Navier-Stokes equations including MCT-type long-lasting memory effects in the stress-strain relations is summarized in Section 3.1. A simple test case is provided by pressure-driven channel flow, where the competition between friction at the rough walls and the driving force leads to inhomogeneous flow. Results from hybrid MCT-LB calculations and from Brownian dynamics (BD) simulations are compared in Section 3.2. The predictive power of a fully microscopic approach to derive constitutive equations is highlighted in non-stationary flow, as it arises, e.g., after the cessation of the driving (force). The structure of the MCT equations admits flow-history dependent residual stresses that persist in the quiescent glass after cessation. The dynamics after cessation of simple shear and in channel flow will be discussed in Section 3.3.

\section{Microscopic approach and mode-coupling theory}

The starting point for a microscopic description of colloidal dispersions is the Smoluchowski equation for the $N$-particle distribution function $P\left(\boldsymbol{r}_{1}, \ldots \boldsymbol{r}_{N}\right)$, which reads $\partial_{t} P=\Omega P$, with the Smoluchowski operator

$$
\Omega(t)=\sum \frac{\partial}{\partial \mathbf{r}_{\mathbf{i}}} D_{i}\left(\mathbf{r}_{\mathbf{i}}\right) \cdot\left(\frac{\partial}{\partial \mathbf{r}_{\mathbf{i}}}-\frac{1}{k_{B} T}\left[\mathbf{F}_{\mathbf{i}}+\mathbf{F}_{\mathbf{i}}^{\text {ext }}\right]\right) .
$$

Here, $\mathbf{F}_{\mathbf{i}}$ are the potential forces describing the interaction among the particles, while $\mathbf{F}_{\mathrm{i}}^{\text {ext }}$ is a (possibly time-dependent) external driving force acting on particle $i$. If particles are moved individually, as in microrheology (see Sect. 2.2), this external force only acts on certain particles (probes), $\mathbf{F}_{\mathrm{i}}^{\text {ext }}=\mathbf{F}_{\mathrm{i}}^{\text {ext }}(t)$, while in macroscopic rheology it is a particle-independent (but possibly space-dependent) driving due to the solvent flow around the colloid, $\mathbf{F}_{\mathrm{i}}^{\text {ext }}=\mathbf{F}^{\text {ext }}\left(\mathbf{r}_{\mathbf{i}}, t\right)$.

The fields of generalized hydrodynamics are obtained from coarse-graining variables that depend on the particles' local contributions [21]. Examples of such variables include the number density $\varrho(\mathbf{r})=\sum_{j=1}^{N} \delta\left(\mathbf{r}-\mathbf{r}_{j}\right)$ or the stress tensor given by the Irving-Kirkwood formula [22]. The external driving in equation (1) induces a hydrodynamic flow field $\mathbf{v}(\mathbf{r}, t)$, which transports material elements at point $\mathbf{r}(t)$ according to $\frac{d}{d s} \mathbf{r}(s)=\mathbf{v}(\mathbf{r}(s), s)$, with $\mathbf{r}(s=t)=\mathbf{r}$. In a system moving with the material element, the dynamical structural processes can be described more easily as advection with the flow field is separated off. Denoting variables in this auxiliary system by a caret, the Smoluchowski equation (1) leads to the nonlinear stress response [23]:

$$
\hat{\boldsymbol{\sigma}}(\mathbf{r}, t)=e_{-}^{-\int_{0}^{t} d s \mathbf{v}(\mathbf{r}(s), s) \cdot \nabla} \boldsymbol{\sigma}(\mathbf{r}, t),
$$

where $e_{-}$denotes the negatively time-ordered matrix exponential and the explicit temporal factor contains the flow advection since flow initiation at $t=0$. In equation $(2 \mathrm{a}), \boldsymbol{\sigma}(\mathbf{r}, t)$ is the macroscopic response to the flow. The temporal evolution of fluctuations in the auxiliary system is given by $\hat{\Omega}^{\dagger}(t)$ which differs from the Smoluchowski operator in equation (1) by the advection with the flow:

$$
\hat{\Omega}^{\dagger}(t)=\Omega_{\mathrm{eq}}^{\dagger}+\sum\left[\Delta D_{i}\left(\boldsymbol{r}_{i}\right)+\frac{1}{\zeta_{0}} \boldsymbol{F}_{i}^{\mathrm{ext}}\left(\boldsymbol{r}_{i}, t\right)-\mathbf{v}(\mathbf{r}(t), t)\right] \cdot \frac{\partial}{\partial \boldsymbol{r}_{i}},
$$


where $\Omega_{\text {eq }}^{\dagger}$ describes the particles' motion in thermal equilibrium, $\zeta_{0}$ is the friction coefficient connected to the diffusivity $D_{0}=k_{B} T / \zeta_{0}$, and $\Delta D_{i}\left(\boldsymbol{r}_{i}\right)$ contains the deviations from $D_{0}$. In general, $\hat{\Omega}^{\dagger}(t)$ thus is a complicated non-local operator which describes the dynamics in response to the inhomogeneous external force field balanced by the motion with the induced flow. Yet, assuming that the inhomogeneities vary smoothly on the scale of the coarse-graining function, a Taylor expansion in $\mathbf{r}_{i}-\mathbf{r}(t)$ can be performed in equation (2b) and the leading term can be canceled by the yet unspecified advective flow field $\mathbf{v}(\mathbf{r}(t), t)$. In linear order in the displacement of the particle positions around the point $\mathbf{r}$, one obtains the following simplified time evolution operator [23]:

$$
\hat{\Omega}^{\dagger}(t) \approx \Omega_{\mathrm{eq}}^{\dagger}+\sum\left[\left(\mathbf{r}_{i}-\mathbf{r}(t)\right) \cdot \boldsymbol{\kappa}(\mathbf{r}, t)\right] \cdot \frac{\partial}{\partial \boldsymbol{r}_{i}} .
$$

A shift of the origin of the particle coordinates by $\mathbf{r}(t)$ renders the auxiliary system homogeneous. It is driven by a velocity gradient field $\boldsymbol{\kappa}(\mathbf{r}, t)=(\nabla \mathbf{v})^{T}$ which is homogeneous on the scale of particle correlations and enters the Smoluchowski operator as a constant tensor. Equation (2) thus leads to a local stress-flow relation, where the coarse grained stress at $\mathbf{r}$ and $t$ is determined by the flow rate within the same coarse-graining volume but at all earlier times. The result is a nonlinear response function, which is obtained from a generalization of Kawasaki and Gunton's nonlinear time-dependent perturbation theory, which they developed to describe hydrodynamic back-flow effects in the viscosity [7]. Equilibrium averaging can be performed. Different from the Newtonian case [24], relaxation in a unique stationary state can be argued for cases described by equation (1) owing to the H-theorem for Fokker-Planck equations $[25]$.

In the homogeneous auxiliary system, the (suitably coarse-grained) stress response $\hat{\boldsymbol{\sigma}}(\mathbf{r}, t)$ in equation (2a) can be related to the Fourier-transformed microscopic potential-stress tensor $\hat{\boldsymbol{\sigma}}(\boldsymbol{q}) \equiv \sum_{i j} \mathrm{r}_{i j} \cdot \mathbf{F}_{i j} \frac{e^{i \boldsymbol{q} \cdot \mathbf{r}_{i}}-e^{i \boldsymbol{q} \cdot \mathbf{r}_{j}}}{i \boldsymbol{q} \cdot \mathbf{r}_{i j}}$. It depends on the position of two particles $\mathbf{r}_{i j}=\mathbf{r}_{i}-\mathbf{r}_{j}$ and on the force $\mathbf{F}_{i j}$ exerted by particle $j$ on particle $i$. Thus, the stress field couples to pair density fluctuations. MCT expresses the response function appearing in equation (2) through transient density correlation functions, assuming that the relaxation of local stresses is dominated by structural relaxation. This results in an approximate constitutive equation applicable close to the glass transition of the form $[8,23]$

$$
\hat{\boldsymbol{\sigma}}(\mathbf{q}=\mathbf{0}, t)=\int_{0}^{t} d t^{\prime} \int \frac{d^{3} k}{(2 \pi)^{3}} \mathcal{V}_{\mathbf{k}}\left[-\partial_{t^{\prime}} \boldsymbol{B}\left(t, t^{\prime}\right)\right] \times \Phi_{\mathbf{k}\left(t, t^{\prime}\right)}^{2}\left(t, t^{\prime},[\boldsymbol{B}]\right),
$$

assuming the system to be in equilibrium at $t=0$. Here, $\mathcal{V}$ is a linear functional given by the equilibrium static structure and the flow history. There appears the Finger tensor $\boldsymbol{B}\left(t, t^{\prime}\right)$, a measure of the deformation between two times $t^{\prime}$ and $t$ that obeys the frame invariance principles of continuum mechanics and is determined by the history of the velocity field and the velocity-gradient tensor $\boldsymbol{\kappa}$. (We drop its parametric dependence on the coarse-grained position $\mathbf{r}$.) The $\Phi_{\mathbf{k}\left(t, t^{\prime}\right)}\left(t, t^{\prime}\right)$ are the transient density correlation functions to a wave vector $\mathbf{k}\left(t, t^{\prime}\right)$ that is advected under flow to the wave vector $\mathbf{k}[8]$. (In the case of simple shear, we denote by $\sigma(t)$ the only non-vanishing element of the deviatoric stress tensor in the following text.)

The evolution equation for the density correlation functions is derived by a projection-operator formalism, and the resulting memory kernel $m\left(t, t^{\prime}, t^{\prime \prime}\right)$ is evaluated by a mode-coupling approximation [8]

$$
\left[\Gamma_{\mathbf{k}}^{-1}\left(t, t^{\prime}\right) \partial_{t}+1\right] \Phi_{\mathbf{k}}\left(t, t^{\prime}\right)+\int_{t^{\prime}}^{t} d t^{\prime \prime} m_{\mathbf{k}}\left(t, t^{\prime \prime}, t^{\prime}\right) \partial_{t^{\prime}} \Phi_{\mathbf{k}}\left(t^{\prime \prime}, t^{\prime}\right)=0
$$




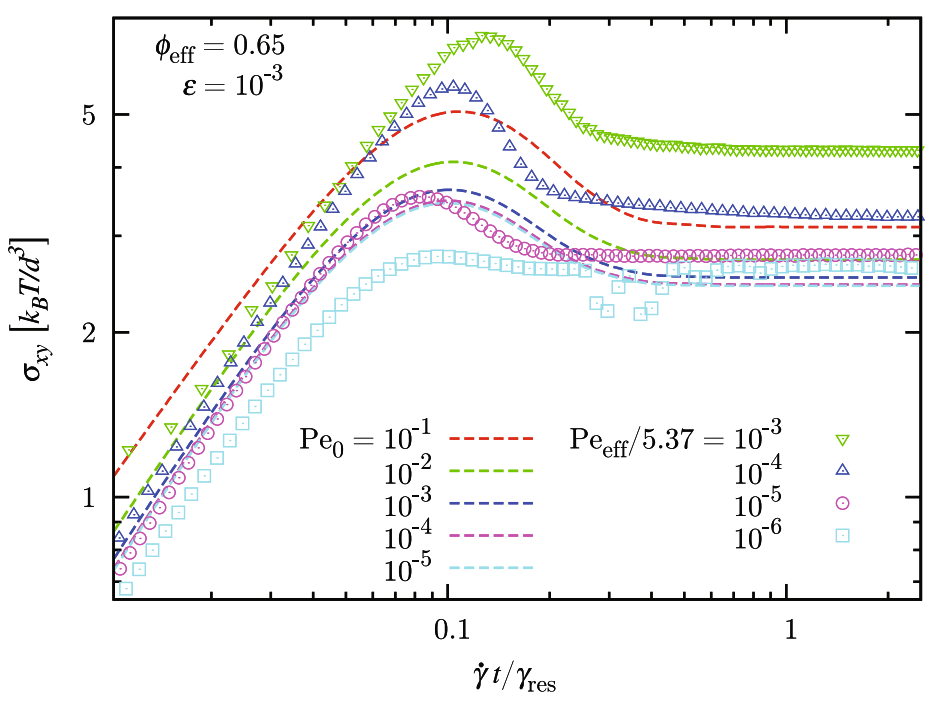

Fig. 1. Stress evolution under start-up shear at constant rate $\dot{\gamma}$ : transient shear stress $\sigma_{x y}$ as a function of the accumulated strain $\dot{\gamma} t$. Solid lines are solutions of full 3D-MCT for a hardsphere (diameter $d=2 R$ ) glassy state; symbols are for experimental data obtained using PNiPAM colloidal hard-sphere-like particles. The applied shear rate is varied, as labeled in terms of the bare Péclet number $\mathrm{Pe}_{0}=\dot{\gamma} d^{2} / D_{0}$. In experiment the measured short time self diffusion coefficient provides Pe $\mathrm{e}_{\mathrm{eff}}$. MCT-ITT strains are rescaled by $\gamma_{\mathrm{res}}=3.4$ and experimental stresses divided by 1.22 ; from reference [28].

$$
m_{\mathbf{q}}\left(t, t^{\prime}, t^{\prime \prime}\right)=\int \frac{d^{3} k}{(2 \pi)^{3}} \mathcal{V}_{\mathbf{q k p}}\left(t, t^{\prime \prime}, t^{\prime}\right) \Phi_{\mathbf{k}\left(t^{\prime \prime}, t^{\prime}\right)}\left(t, t^{\prime \prime}\right) \Phi_{\mathbf{p}\left(t^{\prime \prime}, t^{\prime}\right)}\left(t, t^{\prime \prime}\right)
$$

where translational invariance forces the wavevector $\mathbf{p}$ to be equal to $\mathbf{q}-\mathbf{k}$. The initial decay rate $\Gamma_{\mathbf{k}}\left(t, t^{\prime}\right)=D_{0} k\left(t, t^{\prime}\right)^{2} / S_{k\left(t, t^{\prime}\right)}$ describes local collective diffusion relative to the flow.

Equations (3) and (4a) can be solved numerically in principle, if the equilibrium static structure functions of the liquid under study are known. These encode the interparticle interactions and allow, for example, to study the role of repulsive and attractive interactions in the yielding behavior of amorphous solids [26,27]. However, most studies of MCT have been forced to employ further ad-hoc assumptions regarding the isotropy of density fluctuations or were restricted to the two-dimensional case, because the numerical effort to solve equations (4a) in 3D even in simple stationary flow is enormous. It requires to integrate through the transients, which is called ITT-MCT approach.

Recent progress allowed to solve the ITT-MCT equations of hard-sphere fluids, fully accounting for three-dimensional spatial anisotropy of density fluctuations, in the case of homogeneous startup of simple shear (i.e., a constant shear rate $\dot{\gamma}=(\boldsymbol{\kappa})_{x y}$ assumed to apply instantaneously at all $t>0$, providing a linearly increasing strain $\gamma=\dot{\gamma} t)[27-29]$. The theory provides a quantitative description of the stationary state of glass-forming dispersions under strong constant shearing [28]. The theory captures the fluidization of frozen states and shear-thinning of viscous states observed generically in complex fluids. The microscopic mechanism identified in glass-forming systems is the cage-breaking familiar from the structural relaxation. It is accelerated by the contraction of the cage (structural length) to shorter scales, where irreversible motion is more active. In the case of startup flow, shown in Figure 1, the relaxation of elastic 
energy that is transiently stored in the cages causes a non-monotonic approach to the stationary state: the stress-strain relation $\sigma(\gamma)$ exhibits a characteristic maximum, termed stress overshoot, after the initial regime of elastic deformation $(\sigma \sim \gamma$ for small $\gamma$ ). Stress-strain curves obtained from 3D-MCT can be quantitatively compared to experiment, as shown in Figure 1. The major quantitative error of MCT-ITT concerns the overestimate (by a factor around 3 ) of the strain value where the stress overshoot appears.

From the fully anisotropic MCT, detailed predictions could be obtained for the flow-distorted structure, which is accessible in scattering experiments [29]. While the initial linear increase of $\sigma(\gamma)$ arises from a quadrupolar $(l=2)$ distortion of the local structure [30,31], MCT-ITT identifies hexadecupolar $(l=4)$ distortions as characteristic for plastic decay. They appear around the stress-release signaled by the overshoot, and affect the packing on all neighbour-shells. They thus leave a trace in the hexadecupolar projection of the distorted static structure factor, $\delta S_{q}^{l=4, m=4}$, in phase with the principal peak of the quiescent static structure factor $S_{q}$.

\subsection{Yielding under static load}

A major aim of the new development in MCT applied to driven states is to characterize the differences between force-driven and flow-driven dispersions. The steady state attained under time-independent flow has been extensively discussed within MCT, and has been compared to experiments in model dispersions. For glassy states, MCT predicts a dynamical yield stress: the flow curve $\sigma(\dot{\gamma})$ approaches a nonvanishing constant if the flow rate approaches zero under steady-state conditions, i.e., $\lim _{\dot{\gamma} \rightarrow 0} \sigma(\dot{\gamma})=\sigma_{y}^{\text {dyn }}>0$.

The inverse relations, i.e., the time-dependent strain in response to a constant stress that is instantaneously applied at $t=0$, have been obtained only recently within MCT [33]. The calculation requires the numerically demanding inversion of the functional relation in equations (3) and (4a); it could only be done for a schematic model, where the wavevector-dependence of the density correlation functions is neglected. In this model, equation (3) is replaced by

$$
\boldsymbol{\sigma}(t)=\int_{0}^{t}\left[-\partial_{t^{\prime}} \boldsymbol{B}\left(t, t^{\prime}\right)\right] G\left(t, t^{\prime},[\boldsymbol{B}]\right) d t^{\prime}
$$

and the generalized shear modulus $G\left(t, t^{\prime}\right)$ is evaluated in terms of a single densitycorrelation function $\Phi\left(t, t^{\prime}\right)$ weighted with a strain-dependent coupling vertex, $v_{\sigma}(\gamma)=$ $v_{\sigma}^{*}\left(1-\left(\gamma / \gamma_{*}\right)^{2}\right) \exp \left\{-\left(\gamma / \gamma_{* *}\right)^{2}\right\}[34]$ :

$$
G\left(t, t^{\prime}\right)=v_{\sigma}\left(\gamma\left(t, t^{\prime}\right)\right) \Phi^{2}\left(t, t^{\prime}\right), \text { with } \gamma\left(t, t^{\prime}\right)=\int_{t^{\prime}}^{t} d t^{\prime \prime} \dot{\gamma}\left(t^{\prime \prime}\right)
$$

The transient density correlator $\Phi\left(t, t^{\prime}\right)$ depends on the accumulated strain according to an equation of motion which is obtained from simplifying equation (4a) correspondingly. The inversion proceeds by forward-stepping in time and adjusting $\dot{\gamma}(t)$ iteratively to achieve constant stress; this closely parallels a method to simulate fluids at constant stress [35]. Figure 2 shows typical strain curves for loads around the yield stress which separates states whose asymptotic behavior is either finitely deformed or flowing. Steady-state flow is possible as a long-time asymptote only if $\sigma \geq \sigma_{y}^{\text {dyn }}$, and once homogeneous flow is achieved, the shear rate attained under constant stress corresponds to the inversion of the flow curve [32]. The existence of a maximum in the stress-strain curve, $\sigma_{y}^{\text {stat }}>\sigma_{y}^{\text {dyn }}$, allows for a window of applied 

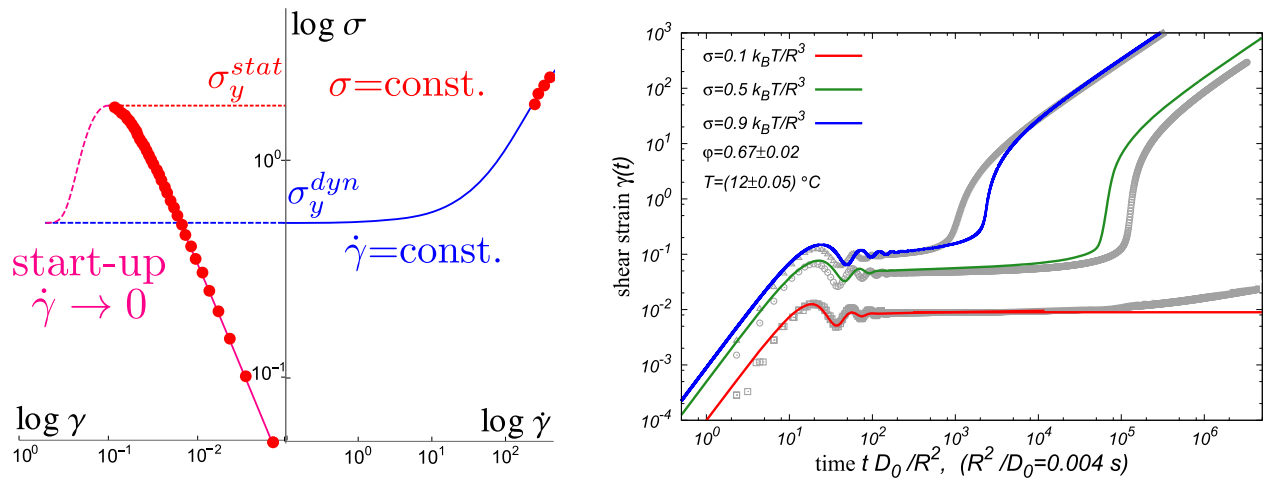

Fig. 2. Schematic-MCT results for the yielding of a glass under applied macroscopic load. Left panel: Red symbols show the asymptotic strain $\gamma(t \rightarrow \infty)$ (left abscissa) and the asymptotic strain rate $\dot{\gamma}(t \rightarrow \infty)$ (right abscissa) under applied stress $\sigma$, obtained from numerical inversion of the constitutive equation. The strain values for $\sigma<\sigma_{y}^{\text {stat }}$ coincide with the stress-strain curve $\sigma(\gamma)$ obtained for infinitely slow startup flow (dashed purple line). For $\sigma>\sigma_{y}^{\text {stat }}$, the shear rates match the flow curve $\sigma(\dot{\gamma})$ of the dynamically yielded flow-driven glass (blue line). (The parameters of the schematic model were adjusted using mesured glass data from Fig. 1, but a larger $\epsilon=0.1$ was chosen.) Right panel: Fits of time-dependent strain $\gamma(t)$ from the schematic model (lines) to experimental data for a glassy PNiPAM microgel dispersion [32] (symbols). Curves correspond to applied stresses $\sigma /\left(k T / R^{3}\right)=0.1,0.5$, and 0.9 (bottom to top, with particle radius $R$ ) and cover a range around the dynamical yield stress $\sigma_{y} /\left(k T / R^{3}\right) \approx 0.4$. From reference [33].

stresses $\sigma \in\left[\sigma_{y}^{\text {dyn }}, \sigma_{y}^{\text {stat }}\right]$ where the initial glass state is deformed but does not yield, while the dynamically yielded state flows. This is confirmed by numerical results for the schematic model (5) [33], shown in the left panel of Figure 2: the asymptotic strain $\gamma(t \rightarrow \infty)$ reached for $\sigma \leq \sigma_{y}^{\text {stat }}$ matches the values corresponding to the stress-strain curve obtained under startup flow in the limit $\dot{\gamma} \rightarrow 0$. The schematic model can be fitted to experimental data obtained on model glass-forming dispersions of polydisperse microgels covering the glass transition at $\phi_{c} \approx 0.64$, as shown in the right panel of Figure 2. (For a discussion of the oscillatory short-time and anomalous long-time transients, see Refs. [32,33].) The comparison is affected by aging phenomena, which are involved in deviations at long times at the lowest stress. The initial glassy state in experiment depends on the preparation history, and hence the strain curves $\gamma(t)$ show aging as a function of the waiting time between preparation and start of the measurement. While Figure 2 shows measurements after a waiting time of $6000 \mathrm{~s}$, additional measurements (not shown) provide the trends when waiting for different periods. With increasing waiting time the data at the lowest stress approach the theoretical line more closely [32,33]. Yet, the limit of infinite waiting-time, which is assumed in MCT, is still not achieved for the lowest curve in Figure 2. The sensitive dependence on preparation protocol and the age of the sample indicates differences to the homogeneously flowing states described by MCT, which could point to transient shear banding effects. These effects are observed with similar characteristics around and above the glass transition density [36-38]. Microscopic theory explaining shear banding focuses on the shear-gradient to density coupling, viz. the feed-back mechanism causing a region with lower shear rate to densify, thereby increasing its viscosity and lowering its flow-rate even more [39]. The sensitive dependence on sample preparation may explain why detailed simulations of equilibrated samples, like considered by MCT, could not observe the coupling [40]. 

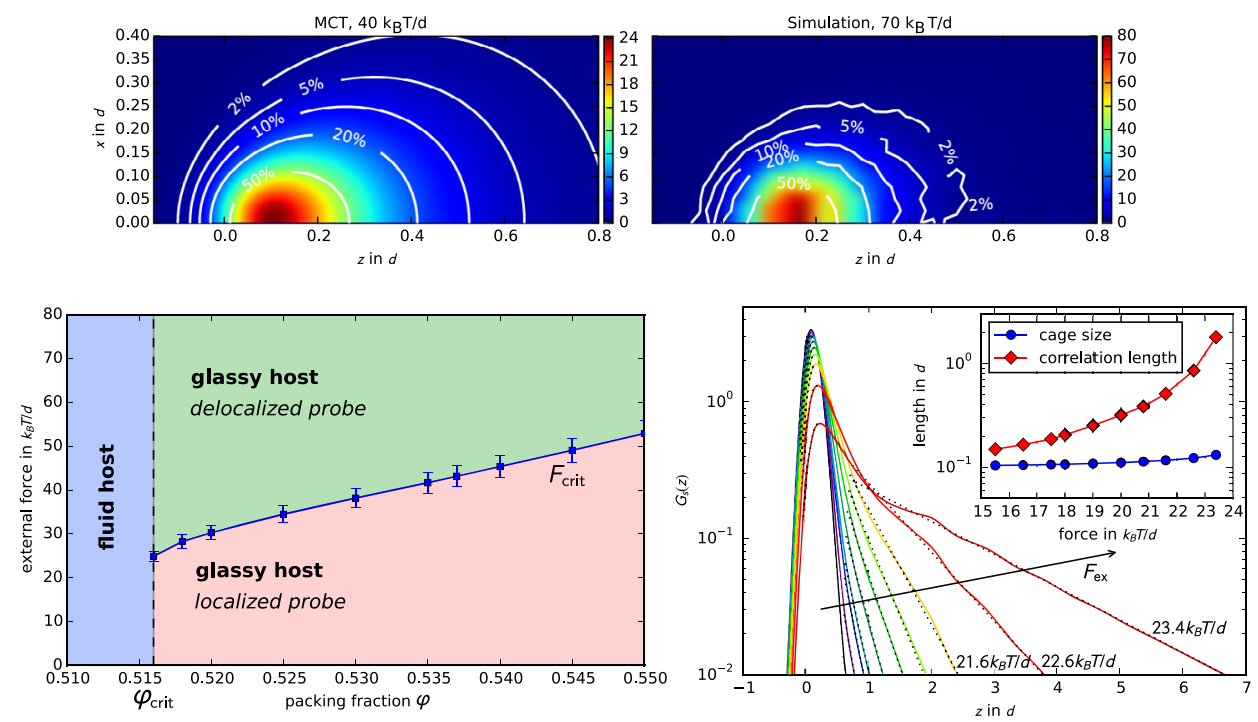

Fig. 3. Force-driven probe particle motion (active microrheology) in glass-forming suspensions. Upper panel: contour plot of the probe-particle density distribution $G_{s}(x, z)$ under applied force (along the $z$-direction) at $t \rightarrow \infty$ for a glassy state of MCT (left), and for a fixed (long) time after application of the force in simulation (right); $d$ is the diameter of the hard spheres. Left panel: MCT state diagram separating delocalized and idealized localized regimes; MCT calculations are done at $\phi=0.537$. Right panel: Long-time limit of the van Hove function $G_{s}(z)$ (integrated over all $x$ ) along the direction of the force for different values of the applied force $F_{\text {ex }}$. Black dashed lines represent exponential decays with a correlation length fitted to the tails of the distributions at long distances. The inset shows the growth of the fitted correlation length (red diamonds) relative to the local cage size (blue circles). From reference [50].

\subsection{Depinning under static force}

The yielding of a glass under applied stress has a microscopic counterpart in the response of a selectively forced probe particle embedded in the glassy host matrix. This response is probed by a technique termed active microrheology that can be a versatile tool to investigate the local rheological properties of complex fluids and glass-forming suspensions [41]. Microrheology also opens the way to the study of the spatial distribution of mechanical parameters in melts on coarser scales [42]. Nonlinear-response effects in microrheology are also discussed in other contributions in this volume [43-45]. Applying a small force to the probe in a glass, a solid-like localized distortion ensues, while above a certain force threshold, the probe undergoes long-ranged delocalized motion with an asymptotically constant velocity [46,47]. The response thus bears certain analogies with depinning (cf. [48]). It is qualitatively different from macroscopic behavior in certain respect: there are characteristic nonanalytic effects even for dilute systems [49].

The extension of MCT to microrheology proceeds along similar lines as the one for shear-driven states. Recently, an improved version of the theory has been derived [50], that accounts for the inherent anisotropy of density fluctuations along and perpendicular to the applied force by introducing parallel relaxation channels for the MCT memory kernel, not unlike previously considered in molecular liquids [51] and in confined geometry [52]. This also cures a finite- $q$ anomaly hidden in the quiescent MCT of the (Lorentz-gas) delocalization transition [53] at finite forces. 
As long as the forced probe remains localized, it explores a deformed cage of neighboring particles. This is reflected in the anisotropic van Hove functions shown in the upper panel of Figure 3. As the force approaches the threshold force from below, this anisotropy becomes more pronounced, and the van Hove functions develop a pronounced tail in the direction of the applied force (lower right panel of Fig. 3). The comparison with Langevin-dynamics simulations testifies that MCT can provide qualitative insight into intermittent dynamics arising from broad probability distributions. The tail of the calculated van Hove function can be well described by an exponential decay, implying a correlation length that grows with increasing force, in broad agreement with the simulation [50]. This correlation length exceeds the cage size (representing the typical length scale responsible for collective glassy arrest) by more than a factor of 10 in the simulations, while theory predicts its divergence at the critical force.

\subsection{Solidity length scale}

Equilibrium correlations in fluids are short-ranged. Elastic strain and stress correlations in solids, on the other hand, are long-ranged according to classical continuum mechanics. While at the familiar first order freezing transition, the creation of longranged static correlations is explained by spontaneous symmetry breaking, at the (kinetic) glass transition only ergodicity is lost, without a clearcut change of symmetry. The emergence of shear rigidity in glass is still a topic of active research (even in linear response $[18,54-57])$. Assuming that correlations at finite frequencies cross over smoothly at a glass transition, the emergence of elasticity requires the buildup of long-lived and long-ranged spatial correlations in supercooled states [58]. While Maxwell anticipated that upon cooling elastic correlations become observable at lower and lower frequencies, it can also be expected that the range of elastic correlations extends further and further. Like the final or Maxwell-relaxation time $\tau$, a solidity length $L$ should exist which diverges at the (idealized) glass transition. For the observation of transverse sound modes in transverse momentum fluctuations, such a length was found in mode-coupling theory calculations [59] and observed in forced Rayleigh scattering [60]. Yet it had been overlooked that it also determines the spatial pattern of strain fluctuations. The development of long-ranged and long-lived strain fields in supercooled liquids has recently attracted interest [61] in the discussion of elastic models for supercooled liquids [62], and the connection to microscopic theory was unclear.

We have investigated far-field strain correlations in liquids within MCT and in video-microscopy experiments on a model two-dimensional glass-forming suspension [63]. Results are shown in Figure 4. The far-field decay of strain correlations follows Eshelby's pattern derived in elasticity theory even though the correlation times far exceed the final relaxation time $\tau$. MCT identifies momentum conservation as the microscopic transport mechanism responsible for the long-ranged strain fields observable inside a region of size $L$ in a liquid. It explains this intriguing observation made in molecular dynamics simulations [61] and in measurements on a colloidal system [63]. The possible consequences of the spatial variations on the viscosity, which has been seen to depend on wavevector $q$ in simulations [64-66], remain to be investigated. While elastoplastic models and simulations [55,67] have established a close link between stress and strain correlations in glass states at low temperatures, the spatial structure of stress correlations in supercooled liquids and their relation to the strain correlations has not been resolved, either. Classical mode-coupling theory [4] and its extensions to flowing states [8] have focused on the global stress and on stresskernels which remain short ranged. Recent extensions of MCT, however, which include 

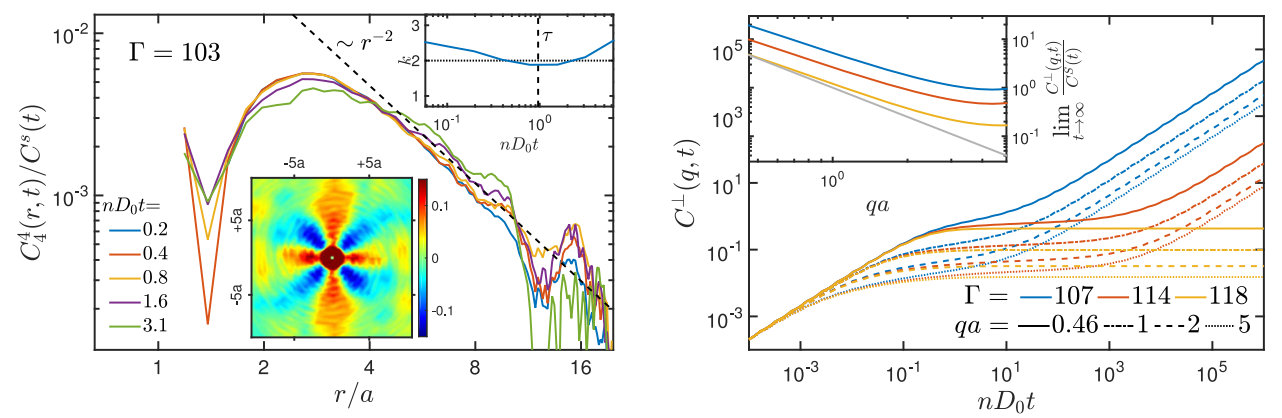

Fig. 4. Strain pattern in supercooled states as measured by video microscopy on layers of colloidal particles and described by MCT; from [63]. Left panel: experimental rescaled strain correlation data for a fluid state (inverse rescaled temperature $\Gamma=103$ far below the glass transition $\Gamma_{c} \approx 200$ ) at different times (see legend); the average particle separation $a$ satisfies $n a^{2}=1$. The radial functions are rescaled to overlap in the far-field power law decay. Main panels show the $1 / r^{k}$-power law decay (dashed black), with exponent $k=2$ varying little with time (upper inset). The contourplots (lower inset) of the long-time limit of $C_{x y}(\mathbf{r}, t) / C_{x y}(\mathbf{r}=0, t)$ illustrate the corresponding $\cos (4 \theta)$-symmetry at $n D_{0} t=3.1>$ $n D_{0} \tau$. Right panel: transversal collective mean-squared displacements $C^{\perp}(q, t)$ from MCT for various wavevectors $q$ as labeled and for two fluid states and one glass one. The inset shows that for wavevectors $q a \ll 1$ the $1 / q^{2}$-behavior (grey solid line) predicted by generalized hydrodynamics is approached at long times; in real space it leads to the far-field power law decay.

coupling of stresses to momentum fluctuations, find that $L$ also determines the stress decay [68]. It remains an important open question to connect these fluid correlations to the elastoplastic modeling developed for glasses at lower temperatures $[55,61,69,70]$.

\subsection{Breakdown of linear response}

The breakdown of linear response in supercooled liquids has recently been connected to the growth of dynamically correlated regions. Bouchaud and Biroli, alluding to the well-understood case of continuous phase transitions among equilibrium phases, argued that nonlinear susceptibility spectra detect the long sought-after domains of cooperative motion in supercooled liquids and record their growth at the glass transition [16]. The response at the third harmonic of the applied sinusoidal perturbation records this signal, which is predicted to diverge as captured in detailed $\alpha$ - and $\beta$-scaling laws at the glass transition [17]. Lacking direct experimental access to them, these predictions were crucial for establishing a route to access the growth of dynamically correlated regions in supercooled liquids experimentally. It enabled nonlinear dielectric spectroscopy to measure the number of dynamically correlated molecules in a number of glass-forming molecular liquids, and to experimentally test the underlying theories going back to fifty year old concepts by Adam and Gibbs [71-73].

In reference [74], we studied the leading nonlinear corrections arising in the stress response of a glass-forming colloidal dispersion driven by sinusoidal shear strain of amplitude $\gamma_{0}$.

$$
\sigma(t)=\gamma_{0} \sum_{n=1}^{3} G_{n}^{\prime}\left(\omega, \gamma_{0}\right) \sin (n \omega t)+\gamma_{0} \sum_{n=1}^{3} G_{n}^{\prime \prime}\left(\omega, \gamma_{0}\right) \cos (n \omega t)+\mathcal{O}\left(\gamma_{0}^{5}\right) .
$$

Compared to the linear response result, which exhibits the same frequency $\omega$ as the external driving, nonlinear corrections in cubic order in strain appear at the 

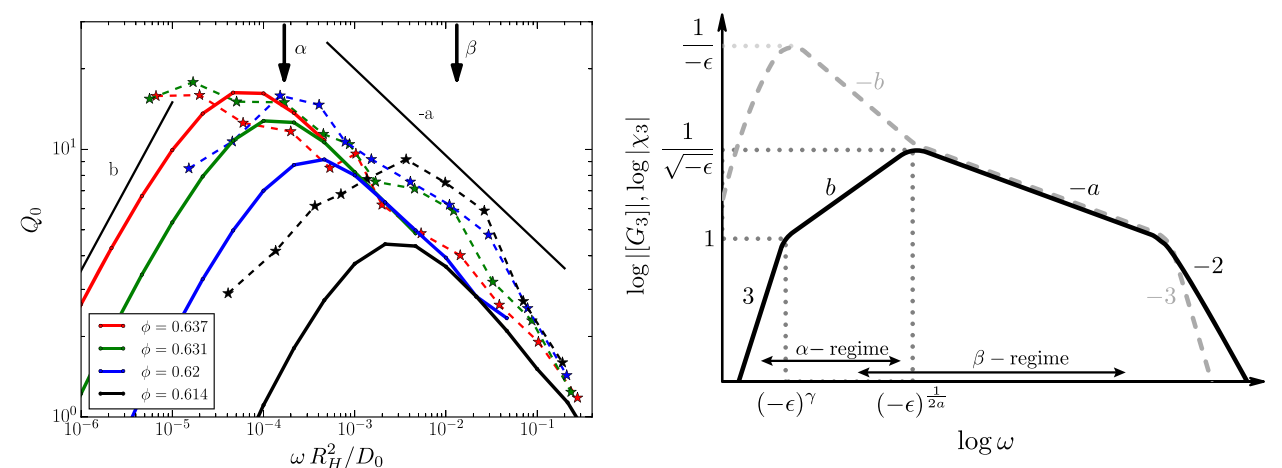

Fig. 5. Leading nonlinear response at higher harmonics under oscillatory shear; from [74]. Left panel: nonlinear response of the third harmonic relative to the linear response $\left(Q_{0}(\omega)=\right.$ $\left.\left|G_{3}\right| /\left|\gamma_{0}^{2} G_{1}\right|\right)$ versus rescaled frequency ( $R_{H}$ is the hydrodynamic radius). Measured data are given as symbols, schematic model calculations within MCT as solid lines with matching colors; the linear response spectra were used to determine the model parameters. Right panel: schematic plot of the scaling of the magnitude of the third-harmonic modulus $G_{3}$ found under shear (black solid line) in comparison to the scaling of the third-harmonic susceptibility $\chi_{3}$ in dielectric systems [17] (dashed gray line); $\varepsilon$ denotes the relative distance to the glass transition.

fundamental mode (viz. $n=1$ ) and at the third harmonic (viz. $n=3$ ). The nonlinear signal at $3 \omega$ is not affected by the linear response and thus can be determined more easily experimentally. Combining high-sensitivity rheological measurements on a well-characterized model glass-former with numerical and theoretical calculations in mode-coupling theory (MCT), we established that the nonlinear response function measured at the third harmonic ( $n=3$ in Eq. (6)) diverges when approaching the glass transition. Figure 5 shows that the results at high frequencies are rather similar to the ones predicted previously for dielectric spectroscopy; however, we find that the divergence is cut off at lower frequencies (viz. in the $\alpha$-relaxation window). The connection of the nonlinear susceptibility to dynamically correlated regions thus differs in the shear-driven case from the cases discussed previously [16]. At present, the sensitivity of the nonlinear response to the kind of perturbing external field is not fully understood. Tarzia et al. [17] considered the nonlinear response to an oscillatory field which, when applied statically, shifts the glass transition locus [75]. Also, they used a generalized fluctuation dissipation relation. In the present case considering shearflow, detailed balance does not hold. Steady shearing melts the glass state of MCT for any shear rate [27]. How these differences result from the microscopic mechanisms tested by the external driving remains to be understood better. While the shearing studies can be rationalized using the cage picture of MCT, the dielectric studies are explained using extended statically and dynamically correlated regions. Further work to relate both approaches would be useful.

\section{Continuum fluid dynamics}

On the continuum scale, the Navier-Stokes equations provide a classical-field-theory approach to the mechanical response of fluids. The relevant fields include the coarsegrained mass density $\rho(\mathbf{r}, \mathbf{t})$ and velocity field $\mathbf{v}(\mathbf{r}, \mathbf{t})$, where $\mathbf{r}$ labels positions in a 
fixed (Eulerian) reference frame. The balance equations of mass and momentum are

$$
\frac{D}{D t} \rho+\rho \nabla \cdot \mathbf{v}=0 \quad \rho \frac{D}{D t} \mathbf{v}=\mathbf{f}^{\mathrm{ex}}-\nabla p+\nabla \cdot \boldsymbol{\sigma}
$$

where we have introduced the material derivative $D / D t \equiv \partial_{t}+\mathbf{v} \cdot \nabla$. Here, $\mathbf{f}^{\text {ex }}$ is an external body-force density, $p$ the (thermodynamic) pressure, and $\boldsymbol{\sigma}$ the Cauchy stress tensor. Constitutive equations such as the one derived from MCT express this latter quantity in terms of the microscopic structural properties of the fluid, and relate it back to the field-theory variables $\rho$ and $\mathbf{v}$, and in particular the velocity gradients $\boldsymbol{\kappa}=(\nabla \mathbf{v})^{T}$.

The schematic-MCT constitutive equation, equation (5a), connects to, and generalizes, a class of well known empirical models for nonlinear rheology. It suggests that $(D / D t) G\left(t, t^{\prime},[\boldsymbol{B}]\right) \approx-f(t) G\left(t, t^{\prime},[\boldsymbol{B}]\right)$ for the long-time dynamics, where $f(t)$ is assumed to depend on the flow fields only instantaneously. This leads to

$$
\frac{D}{D t} \boldsymbol{\sigma}-\boldsymbol{\kappa} \cdot \boldsymbol{\sigma}-\boldsymbol{\sigma} \cdot \boldsymbol{\kappa}^{T}=\stackrel{\nabla}{\boldsymbol{\sigma}}=G_{\infty} \boldsymbol{D}-\boldsymbol{f} \boldsymbol{\sigma}
$$

with $\boldsymbol{D}=\boldsymbol{\kappa}+\boldsymbol{\kappa}^{T}$. The left-hand side of this equation is known as the upper-convected derivative of the stress tensor [76]; it ensures material objectivity on the continuum scale. It arises in the MCT-approach from going to the auxiliary system in equation (2), where the frame invariant finger tensor encodes the deformation. The (spaceand time-dependent) quantity $f$ defines a "fluidity" field. Various recent mesoscopic approaches to glassy rheology aim at modeling an evolution equation for the fluidity [77-82]. For the linear response of a viscoelastic fluid, $f \equiv 1 / \tau$, and equation (8) defines the so-called upper-convected Maxwell model [6]. A class of phenomenological models includes shear-thinning effects by assuming $f$ to be a local functional of the velocity gradients; these are known as White-Metzner models [6]. If one sets

$$
f(\mathbf{r}, \mathbf{t})=1 / \tau+|\dot{\gamma}(\mathbf{r}, \mathbf{t})| / \gamma_{c},
$$

one obtains a White-Metzner model that reduces in steady flow to a generalized Maxwell model. The latter was introduced earlier as a simple model to capture the qualitative phenomena of the MCT phenomenology of steady-state shear-thinning flow close to the glass transition $[83,84]$. In this model, the local flow rate $|\dot{\gamma}|$ is taken to be a suitable scalar invariant of the velocity-gradient tensor $\boldsymbol{\kappa}$, while $\gamma_{c}$ is a parameter that sets the typical strain at which neigbor-cages break (closely related to the position of the stress overshoot as discussed above).

We are concerned here with the limit of low Mach number, i.e., all relevant velocities shall be much smaller than the speed of sound. In this limit, the use of the constitutive equation derived from MCT for colloidal suspensions can be justified. Also, the hydrodynamic flow shall be assumed nearly incompressible, $\nabla \cdot \mathbf{v} \approx 0$. We are also interested in the limit of low Reynolds number, $R e \ll 1$.

In the case of Newtonian fluids, flows at large- $R e$ are prone to instabilities driven by inertial effects, generally leading to turbulence. Low-Re flows of non-Newtonian fluids with curved flow-lines can exhibit phenomenologically similar non-inertial flow instabilities. So-called elastic turbulence has been observed in a number of complex fluids such as worm-like micelles or polymer solutions [85]. Material elasticity in this case drives a linear instability of the flow: in a cylindrical Couette flow, the normal component of the stress $\sigma_{\theta \theta}$ couples to the radial velocity component, and thus a radial velocity perturbation can be amplified. The description of this instability is a challenge for developing extensions of MCT to inhomogeneous flows [23].

Interestingly, shear thinning strongly suppresses the elastic instability. In stationary Taylor-Couette flow of the White-Metzner model given by equation (9), all 
perturbations of the velocity field are linearly stable for physically plausible values of the strain $\gamma_{c}$ required to break nearest-neighbor cages [23]. The predicted stabilizing effect was recently verified in serpentine microflows of well characterized dilute polymer solutions [86]. (But see Ref. [81] for effects of another type of visco-elastic instability in flow through straight channels of fluids with different shear-thinning characteristics.)

\subsection{Hybrid lattice-Boltzmann algorithm}

To solve the Navier-Stokes equations for a Newtonian fluid in the low Mach number limit, the Lattice Boltzmann (LB) method is a versatile and efficient method $[87,88]$. On a suitably chosen spatial lattice, one introduces local densities $n_{i}(\mathbf{r}, \mathbf{t})$ corresponding to discrete velocity vectors $\mathbf{c}_{i}(i=0, \ldots 8$ for the most common $2 \mathrm{D}$ lattice with velocities pointing to nearest and next-to-nearest lattice sites). The physical fields are determined by the moments of the local densities formed with the lattice velocities. The LB simulation evolves the lattice populations by a sequence of streaming and collision steps:

$$
n_{i}\left(\mathbf{r}+\mathbf{c}_{i} \delta t, t+\delta t\right)=n_{i}(\mathbf{r}, \mathbf{t})+\Delta[n(\mathbf{r}, \mathbf{t})]+F_{i} .
$$

During the streaming step, the lattice densities are advected according to the corresponding lattice velocity; during the collision step, the densities are relaxed locally (by a linear operator $\Delta$ acting on each lattice node) towards an equilibrium form $n_{i}^{\text {eq }}$ that depends (only) on the fields $\rho$ and $\mathbf{v}$. The latter is chosen such as to ensure that in the continuum limit, the physical fields obtained from LB approach the solutions of the Navier-Stokes equation (up to higher-order corrections in the lattice velocities).

Non-Newtonian effects can be incorporated by including an extra forcing term $F_{i}$ during the LB collision step. A Chapman-Enskog analysis fixes the form of this forcing term, given an arbitrary constitutive equation for the non-Newtonian stresses [89]: there enters the contraction of the traceless stress tensor with the lattice velocities, $F_{i} \propto \mathbf{c}_{i} \mathbf{c}_{i}: \overline{\boldsymbol{\sigma}}$. (Throughout, we implicitly assume a background Newtonian viscosity, as is appropriate for colloidal suspensions.)

In order to couple to the Navier-Stokes equations history-dependent nonlinear response entering the constitutive equation, the $\mathrm{LB}$ simulation needs to be coupled to an integral-equation solver capable of solving equation (3) (or equations of similar type). Such a hybrid-LB algorithm extends each spatial LB lattice node by a time axis in order to store the history of local flow [90]; for the inclusion of MCT, further memory is needed to store the local correlation functions and memory kernels needed to integrate equation (3) [91]. In order to limit the computational demand, it is assumed that the nonlinear response function becomes slowly varying in time once the time interval $t-t^{\prime}$ becomes large; this assumption is inherent to all numerical approaches to MCT and potentially limits the evaluation of the response to external drive that varies slowly over arbitrarily large time intervals. For a description of the adaptive time-domain grids, see references [90,91].

A central aspect of the hybrid-LB algorithm is the capability to store the deformation history in such a way as to enable the calculation of material-objective stresses. One common approach is to store the values of the Finger tensor $\boldsymbol{B}\left(t, t^{\prime}\right)$ on a grid of past times $t^{\prime}<t$; it can be obtained by solving the defining differential equation $\stackrel{\nabla}{\boldsymbol{B}}\left(t, t^{\prime}\right)=\mathbf{0}$ for all desired fixed $t^{\prime}$. In the context of finite-element or finite-volume methods, this approach is known as the deformation-field method [92-94] (where it is, however, so far restricted to constitutive equations where the response function is time-translational invariant). Formally, the Finger tensor can be expressed in terms 

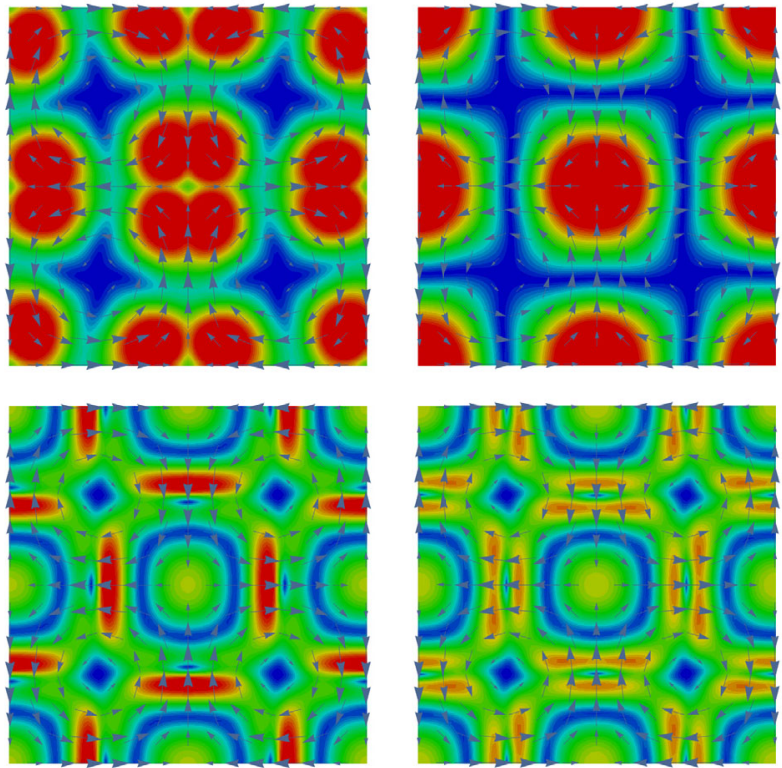

Fig. 6. Stress distribution after cessation of Taylor-Green vortex flow, in the upper-convected Maxwell model (top panels) and a glassy White-Metzner model (bottom panels) with relaxation time $\tau / \tau_{0}=100$. The stress magnitude $\|\boldsymbol{\sigma}\|$ is shown (blue: low; red: high values), for a time $t / \tau_{0}=5.6$ after cessation. Left panels show the full solution, right panels the solution without stress advection (see text) [82]. Arrows indicate the initial velocity field at $t=0$.

of a deformation tensor, $\boldsymbol{B}\left(t, t^{\prime}\right)=\boldsymbol{E}\left(t, t^{\prime}\right) \cdot \boldsymbol{E}\left(t, t^{\prime}\right)^{T}$. The deformation tensor is the solution of $(D / D t) \boldsymbol{E}\left(t, t^{\prime}\right)=\boldsymbol{\kappa}(t) \cdot \boldsymbol{E}\left(t, t^{\prime}\right)$, written as the time-ordered exponential $\boldsymbol{E}\left(t, t^{\prime}\right)=\exp _{+}\left[\int_{t^{\prime}}^{t}(\boldsymbol{\kappa}(s)-\mathbf{v}(s) \cdot \nabla) d s\right] \mathbf{1}$. In the simulation, it can be constructed by approximately evaluating $\boldsymbol{E}\left(t, t^{\prime}\right) \approx \exp [\delta t(\boldsymbol{\kappa}(t)-\mathbf{v}(t) \cdot \nabla)] \cdot \boldsymbol{E}\left(t-\delta t, t^{\prime}\right)$. In the case of advection-free flow where $\mathbf{v}(t) \cdot \nabla \equiv 0$, these expressions simplify considerably and allow for an efficient integration of the flow history [91].

A simple test case for the effects of stress advection is Taylor-Green vortex flow: by a suitable body-force density one initializes a velocity field of the form $v_{x}(x, y, 0)=$ $v_{0} \sin x \cos y$ and $v_{y}(x, y, 0)=-v_{0} \cos x \sin y$ at $t=0$. After that, the decay of the velocity and stress fields are observed. Figure 6 shows the stress magnitude $\|\boldsymbol{\sigma}(x, y, t)\|$ at a fixed time after cessation for a linear-response and a shear-thinning model of glassy rheology according to equation (9) with relaxation time $\tau / \tau_{0}=100$, denoting the Brownian time as $\tau_{0}=d^{2} / D_{0}$. Results including and excluding stress advection in the constitutive equation are compared. The flow obeys $P_{e} \ll 1$, and the velocity fields are similar in both cases. Even so, noticeable differences appear in the stresses. Shear thinning reduces the effect of stress advection (unless the velocity field has a constant offset): in Figure 6, the results for the White-Metzner model with and without stress advection term are much more similar than for the upper convected Maxwell model. Typically, advection is stronger in regions that also have stronger local shear rate, and there, flow-induced stress relaxation becomes more effective.

\subsection{Channel flow}

Pressure-driven flow through micro-channels provides a test for spatial correlations in driven complex fluids as the shear rate and thus the induced local structural relaxation 
naturally varies across the channel [95]. Already in the quiescent state, a rich variation of the dynamics arises along and perpendicular to the rough walls [96-98]. Preparing rough walls by freezing in layers of particles in equilibrated samples connects to the topic of the glass transition in the presence of pinned particles [99,100]. Under flow quite universally a central plug develops where the soft glassy material moves uniformly while the shear is localized to narrow regions close to the walls $[101,102]$. At lower colloid concentrations, particle migration [103] and marked differences of the particle motilities can be observed by microscopy in hard sphere suspensions [104].

The generalized nonlinear Maxwell model, equations (7) to (9), allows an analytical solution for the steady-state channel flow, once the flow is assumed to be translational-invariant along the channel [90]. The model assumes a local relation between stress and flow-rate. From the Navier-Stokes equation, the shear stress $\sigma_{x y}$ vanishes in the center of the channel, and a central region emerges where $\sigma_{x y}$ remains below the critical yield stress that emerges at the glass transition. In this region, the local shear rate vanishes as $1 / \tau$ for $\tau \rightarrow \infty$. There appears a normal-stress difference $\sigma_{x x}-\sigma_{y y}$ with a parabolic minimum in the center of the channel, indicating an anisotropic contribution to the pressure that acts inwards from the sheared regions close to the channel walls. This phenomenology is consistent with the more involved schematic-MCT model given in equation (5). The latter has been solved using our hybrid-LB-MCT algorithm (setting $v_{\sigma}=$ const.); the resulting steady-state quantities are shown in Figure 7 (leftmost panel). In comparison to the generalized nonlinear Maxwell model (solid lines in the figure), the schematic-MCT constitutive equation predicts the normal-stress difference to vary over a wider cross-section of the channel. Note that the LB algorithm solves weakly incompressible flow. Therefore, an isotropic stress contribution arising from the non-Newtonian flow causes a corresponding density change $\delta \rho$ that is calculated from the linearized equation of state, $p \sim c_{s}^{2} \delta \rho$ (where an overall constant is irrelevant in incompressible flow). The LB-MCT calculation thus suggests a (weak) increase of the local density in the plug region.

Event-driven Brownian-dynamics (ED-BD) simulations [106] of a binary glassforming $2 \mathrm{D}$ hard-disk mixture have been performed to test the model [105]. The system is a well-known model glass former that undergoes a MCT transition at packing fraction $\phi_{c} \approx 0.795[107,108]$ whose homogeneous nonlinear rheology has been studied in detail (see, e.g., Refs. $[28,109]$ ). To mimic the no-slip boundary conditions used in the continuum-mechanics description, rough walls were implemented by freezing layers of particles in a previously equilibrated configuration. A pressure gradient was implemented through a body force acting on the particles [105]. The simulations confirm the explanation of regions with (asymptotically) negligible flow rate based on the criterion involving the dynamic yield stress [90,102]. As shown in Figure 7 (left and center panels), LB-MCT and the BD simulations agree qualitatively for the velocity, shear-rate and stress tensor profiles. In the BD simulations, the small increase of the local density is observed in the center of the channel, which was anticipated from the increased pressure in the LB-MCT model, as explained above. The nonlinear Maxwell model can be mapped onto the BD simulations at not too large forces where the observed shear rates correspond to externally imposed relaxation times which are larger than the intrinsic short time dynamics [105]. The validity of the theoretical description in the limit of a separation of long-time structural or driven dynamics from (diffusive) short time dynamics supports MCT's prediction of dynamical scaling laws (called $\alpha$ - and $\beta$ - process). Apparently, MCT provides the basis for the rational prediction of macroscopic constitutive equations (viz. the nonlinear Maxwell model suggested by White and Metzner which agrees with an $\alpha$-scaling law by MCT for driven fluids), which rationalize particle based simulations of viscoelastic fluids. For the channel flow, this verifies that a local theory, connecting stress and strain at the 

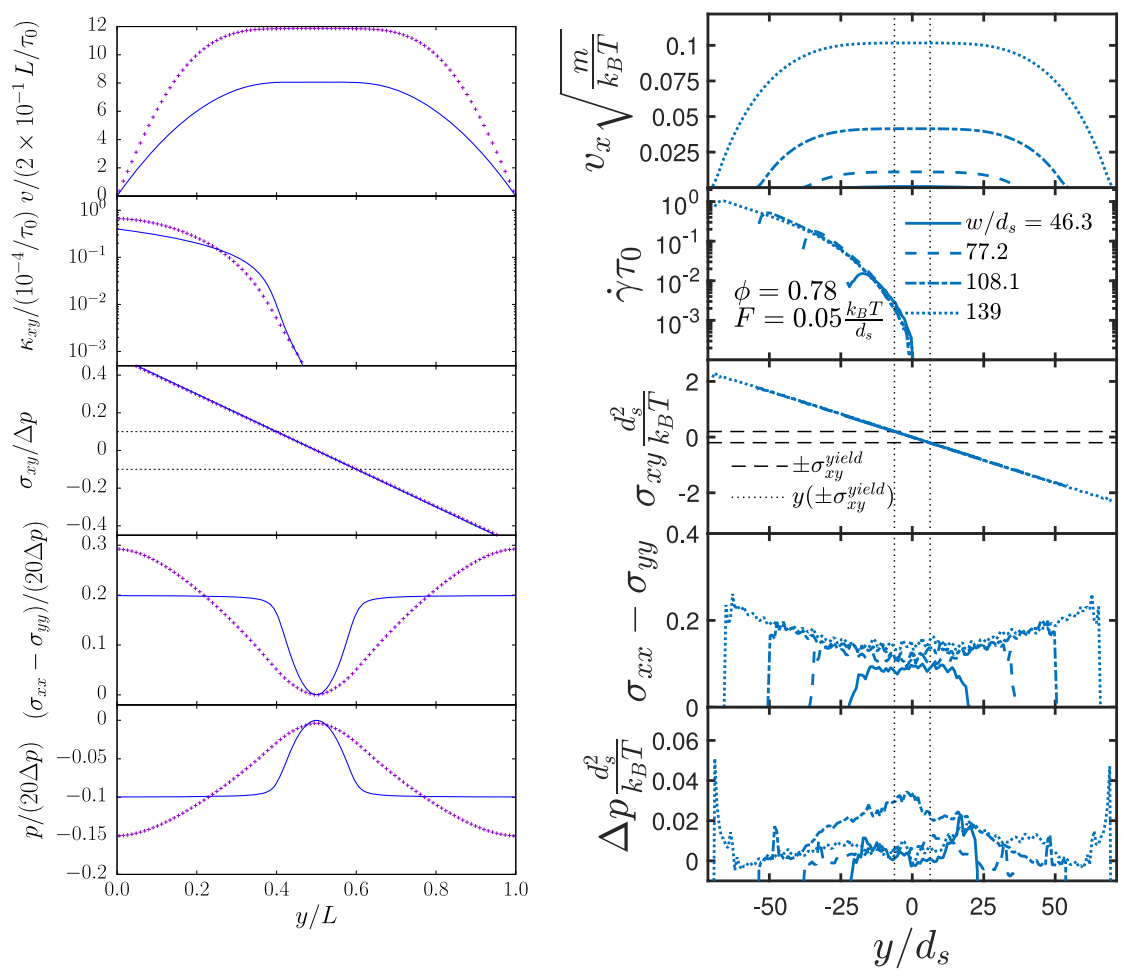

Fig. 7. Pressure-driven flow of glassy fluids through a rough microchannel. Left panel: velocity profile $v_{x}(y)$, shear rate $\dot{\gamma}=\kappa_{x y}$, shear stress $\sigma_{x y}$, normal-stress difference $\sigma_{x x}-$ $\sigma_{y y}$ and pressure $p$ (top to bottom) from a hybrid-LB-MCT calculation using a schematicmodel state close to the glass transition (crosses). Solid lines are analytical solutions of the generalized nonlinear Maxwell model for reference. Horizontal dashed lines mark the yield stress of the model at the glass transition. Data from reference [91]. Right panel: corresponding results from $\mathrm{BD}$ simulations using a $2 \mathrm{D}$ glass-forming hard-sphere mixture in channels of different widths as given in the legend; see there also for packing fraction and force. Data from reference [105].

same points in space (but incorporating memory) explains plug flow in viscoelastic states qualitatively. The hybrid-LB-MCT calculations in particular give a much improved description of the normal-stress differences, compared to the generalized nonlinear Maxwell model. Non-local effects arise in the BD simulations at higher packing fractions [105], which have previously been discussed in channel-flow geometry $[80,81,95,110,111]$. Ongoing work aims to establish this link within microscopic $\mathrm{MCT}$, and to connect the effect to the far-field strain correlations discussed above.

\subsection{Cessation dynamics}

To explore the qualitative effects predicted by the MCT constitutive equation, we turn to non-stationary flow. While the symmetry principles behind continuum mechanics are powerful principles to restrict the tensorial structure of the equations, they give little indication towards their time-dependence. MCT is based on an exact evolution equation for density correlation functions, equation (4a), derived from nonlinear-response theory. The time-dependence of the memory kernel appearing in 


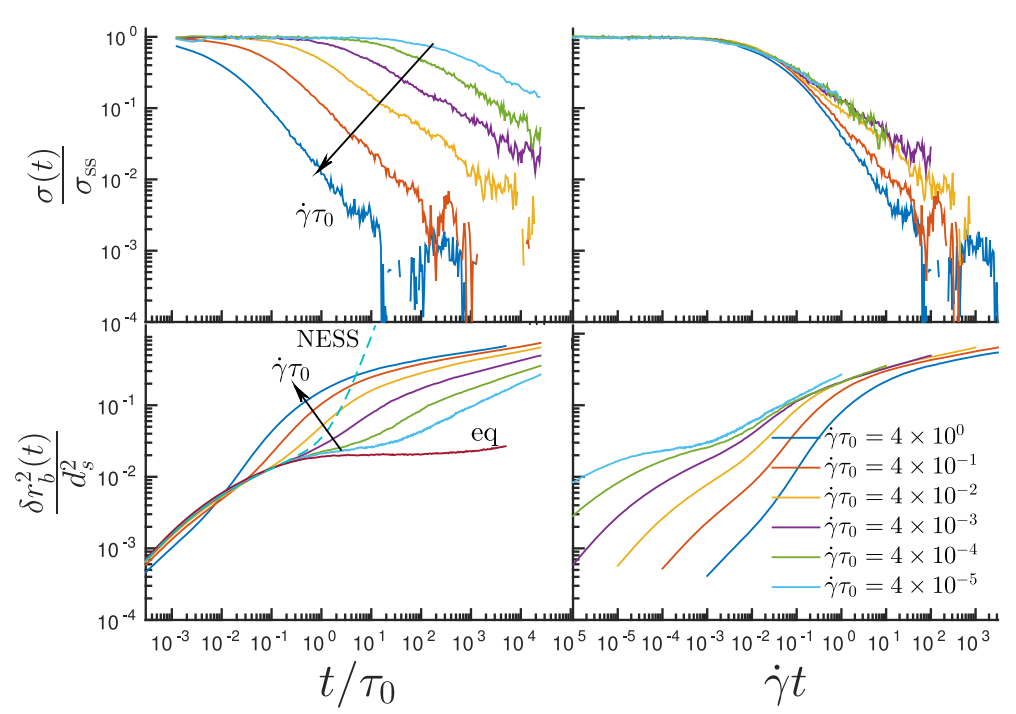

Fig. 8. Upper panels: stress relaxation after cessation of shear flow from the nonequilibrium stationary state at a shear-rate $\dot{\gamma}$ switched off at $t=0$ (adapted from Ref. [112]). The lower panels show the evolution of mean squared displacement with time after switch-off. The right panels show the same data as functions of rescaled time $\dot{\gamma} t$.

this equation is rather subtle and qualitatively goes beyond the constitutive equations that are typically employed in applied rheology. This affects in particular the transient flow behavior after cessation.

Within MCT, the stress relaxation after cessation of homogeneous simple-shear flow with prescribed shear rate $\dot{\gamma}$ has been studied [113]; it considers the stress at $t>0$ with the shearing protocol $\dot{\gamma}(t<0)=\dot{\gamma}$ and $\dot{\gamma}(t>0)=0$. In the glass, MCT describes temporal memory that can stretch back arbitrarily far in time, and as a result, stresses do not relax to zero, but to a finite residual-stress value that depends on the initial flow rate. These residual stresses have been confirmed in molecular-dynamics and BD simulations and different experiments on various colloidal hard-sphere-like systems $[112,113]$. Phenomenologically, residual stresses are known to exist in quenched glasses; they are exploited technologically to tune the macroscopic mechanical performance of materials produced from the melt, e.g., in safety glasses. The extension of MCT to nonlinear rheology provides a first avenue to study the microscopic origins of residual stresses.

The local particle dynamics that is responsible for residual stresses has been addressed in simulations in terms of the mean-squared displacement (MSD). Results from BD simulations are shown in Figure 8. The lower panels show the MSD during the relaxation of the system from the nonequilibrium steady state (NESS). The MSD first follows the curve describing the steady state dynamics up to a time $\dot{\gamma} t \ll 1$ retaining the memory of the previous shear flow at rate $\dot{\gamma}$. After that, it increases and crosses over to a broad window of sub-diffusive growth. The data suggests that in the ideal glass, the MSD will approach a second plateau. Note that the length scale associated with the first, or quiescent plateau, $\delta r^{2} \approx 0.02 d^{2}$, is linked to the Lindemann lengthscale of localized in-cage dynamics, $r_{\text {loc }} / d=\sqrt{\delta r^{2} / 4 d^{2}} \approx 7 \%$ in our case. The second plateau, $\delta r^{2} \approx d^{2}$, corresponds to particle movements of about $50 \%$ of the diameter, much larger than Lindemann's criterion for melting. Similar MSD have been found in aging after thermodynamic quenches for colloidal suspensions [114] and for computer simulations $[115,116]$. A second plateau has been noted in reference [113] 

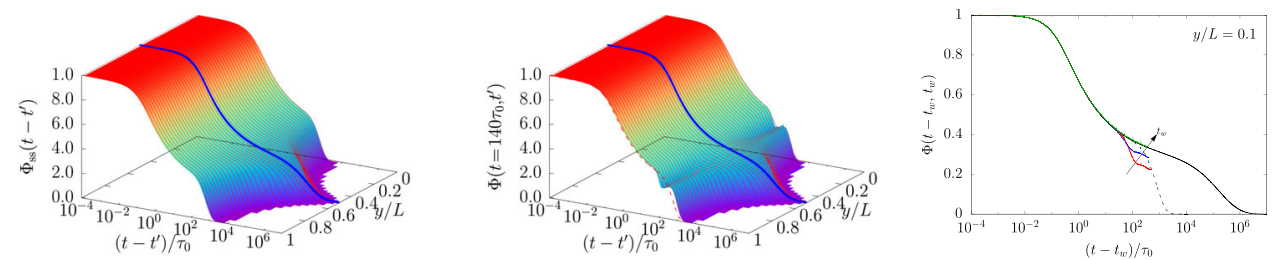

Fig. 9. Relaxation of density correlation functions $\Phi\left(t, t^{\prime}\right)$ during cessation of pressure-driven planar channel flow, obtained from hybrid-LB-MCT simulations for a fluid state close to the glass transition. Left: steady-state correlation functions $\Phi_{\mathrm{NESS}}\left(t-t^{\prime}\right)$ as a function of time difference and transversal position across the channel. A thick blue line indicates the quiescent equilibrium correlation function. Center: transient correlation functions $\Phi\left(t, t^{\prime}\right)$ at fixed $t=140 \tau_{0}$ after removal of the driving pressure gradient at $t=0$. Right: waiting-time dependent correlation functions $\Phi\left(t-t_{w}, t_{w}\right)$ at fixed $t_{w}>0$ up to $t-t_{w}=500 \tau_{0}$ and fixed transversal position $y / L=0.1$. A dashed line shows the steady-state correlation function, the solid line is the quiescent equilibrium correlator. Data from reference [91].

in the waiting-time dependent MSD from molecular dynamics simulations and measured for colloidal suspensions through confocal microscopy. It has been interpreted as signalling the size of fluidized regions. The present BD simulations extend this finding from fluid to states above the MCT glass transition.

If flow cessation is studied from a NESS that is driven by an applied external force, the assumption that the local shear rate goes to zero instantaneously, $\dot{\gamma}(t)=0$ for $t>0$, may not be justified. In this case, the combined MCT-LB approach allows to study the interplay of microscopic stress relaxation and macroscopic flow cessation. After cessation of pressure-driven planar channel flow, LB-MCT predicts an oscillatory decay of both the velocity profile and the stresses towards zero [91].

The oscillatory macroscopic stress relaxation can be linked to the microscopic dynamics in terms of the transient density correlation functions [91]; LB-MCT results are shown in Figure 9. In the stationary state prior to cessation (left panel), the decay of the correlation functions reflects the local shear rate: in the center of the channel, the correlation functions approach the quiescent equilibrium one, while close to the channel walls, the relaxation is much faster owing to the increase in the local Péclet number. During cessation, the system is in a non-stationary state, and hence the correlation functions depend on both time arguments separately. A study of the stresses at time $t$ after cessation suggests to consider the correlation functions $\Phi\left(t, t^{\prime}\right)$ at fixed first time argument; the corresponding data is shown in the center panel of Figure 9. Around $t^{\prime}=0$ (flow cessation), a pronounced oscillation is seen in the decay of $\Phi\left(t, t^{\prime}\right)$ close to the channel walls. This indicates that locally, density correlations can be reinstated much like in large-amplitude oscillatory shear. A more common way to discuss non-stationary correlation functions is for fixed second time argument, i.e., for fixed waiting time $t_{w}=t^{\prime}$. These quantities are shown in the right panel of Figure 9 for a fixed transversal position close to the channel wall. Since the numerical effort for the LB-MCT calculations is considerate, these functions could only be obtained for $t-t_{w}<500 \tau_{0}$. For small $t_{w}$, the correlation functions indicate a decay with a second plateau less than the typical cageing plateau known from the quiescent glassy dynamics. Qualitatively, this is reminiscent of the second plateau discussed above for the MSD after cessation of steady shear. For the channel flow, this second plateau vanishes with increasing $t_{w}$.

Note that the case shown in Figure 9 corresponds to a liquid-like quiescent state; hence, no residual stresses exist after cessation. However, LB-MCT can be used to study how residual stresses can arise in situations where after cessation of the driving force, the velocity gradients do not instantaneously decay to zero but evolve on the same scale as the stresses. 


\section{Conclusions}

We have reviewed recent progress in understanding inhomogeneous flow of forcedriven colloidal suspensions close to the glass transition on the basis of ITT and MCT. In linear response, it does not matter whether a system is driven by a force or by its conjugate generalized displacement. This is no longer true in nonlinear response. In glasses, the existence of a yield stress causes the response under applied force to be much more intricate, compared to the case of fluidized states. In particular, applied forces close to the yield stress for thermodynamic parameters close to the glass transition tend to amplify spatial inhomogeneities. Understanding the interplay between temporal and spatial non-localities remains an ongoing endeavor when extending MCT to inhomogeneous driving building on the reviewed progress.

Yielding of glassy states and shear-thinning of homogeneous viscoelastic states appears well captured by MCT-ITT with the force-induced breaking of cooperative local cages as the dominant effect. A unique NESS is approached independently of the external driving, except, possibly, in solid states at loads around the yield stress. The NESS is characterized by divergent nonlinear susceptibilities, e.g., at higher harmonics of the periodic driving. Yet flow does not couple to cooperatively-rearranging domains and the nonlinear spectra are suppressed at low frequencies. Elastic correlations in the solid state are long-ranged and the depinning of forced particles suggests that important spatial correlations build up in the deformed but not yet yielded soft solid. This strongly points to a link of MCT's cage-breaking in supercooled states to elastoplastic low-temperature phenomena which are understood as arising from localized plastic excitations coupled by long-ranged elastic interactions $[69,70]$.

Planar channel flow was investigated as a simple test case for a situation where the flow rate is inhomogeneous. Combining MCT with a continuum-fluid dynamics lattice Boltzmann (LB) algorithm, it was demonstrated that MCT captures the known phenomenology of the stationary flow of yield-stress fluids (at not too low temperatures and below a jamming transition). In the transient dynamics after the cessation of flow, the intricate time-dependence encoded in the MCT-based material law becomes important. It allows to describe genuine nonlinear-response phenomena such as residual stresses and material properties that depend on the past flow history.

The recent development, both of MCT as a microscopic theory for the nonlinear response of glass-forming suspensions as well as of LB as a tool to investigate the effects of non-Newtonian rheology on the macroscopic continuum scale, provides a promising new starting point for a systematic development of material laws that capture both temporally and spatially non-local effects, in a way that is consistent with the fundamental principles of nonequilibrium statistical physics.

The work reviewed here was supported by the Deutsche Forschungsgemeinschaft (DFG) through the Research Unit FOR1394 "Nonlinear Response to Probe Vitrification", project P3. We thank its members for many fruitful discussions. Th.V. thanks M.E. Cates, B. Dünweg and T. Krüger for helpful discussions regarding the lattice Boltzmann algorithm. S.F. and M.F. thank F. Varnik for helpful discussions regarding the BD channel flow simulations. Finally, the authors cordially thank their collaborators of the reviewed joint project.

\section{References}

1. J.C. Maxwell, Philos. Trans. R. Soc. London 157, 49 (1867)

2. E. Leutheusser, Phys. Rev. A 29, 2765 (1984)

3. U. Bengtzelius, W. Götze, A. Sjölander, J. Phys. C: Solid State Phys. 17, 5915 (1984)

4. W. Götze, Complex Dynamics of Glass-Forming Liquids, A Mode-Coupling Theory (Oxford University Press, 2009) 
5. G.L. Hunter, E.R. Weeks, Rep. Prog. Phys. 75, 066501 (2012)

6. R.G. Larson, The Structure and Rheology of Complex Fluids (Oxford University Press, New York, 1999)

7. K. Kawasaki, J.D. Gunton, Phys. Rev. A 8, 2048 (1973)

8. J.M. Brader, M.E. Cates, M. Fuchs, Phys. Rev. E 86, 021403 (2012)

9. K. Suzuki, H. Hayakawa, Phys. Rev. E 87, 012304 (2013)

10. W.T. Kranz, M. Sperl, A. Zippelius, Phys. Rev. E 87, 022207 (2013)

11. Th. Voigtmann, Curr. Opin. Colloid Interf. Sci. 19, 549 (2014)

12. A.A. Aerov, M. Krüger, J. Chem. Phys. 140, 094701 (2014)

13. A.A. Aerov, M. Krüger, Phys. Rev. E 92, 042301 (2015)

14. P. Hopkins, A. Fortini, A.J. Archer, M. Schmidt, J. Chem. Phys. 133, 224505 (2010)

15. P. Krinninger, M. Schmidt, J.M. Brader, Phys. Rev. Lett. 117, 208003 (2016)

16. J.P. Bouchaud, G. Biroli, Phys. Rev. B 6, 064204 (2005)

17. M. Tarzia, G. Biroli, A. Lefèvre, J.P. Bouchaud, J. Chem. Phys. 132, 054501 (2010)

18. G. Szamel, E. Flenner, Phys. Rev. Lett. 107, 105505 (2011)

19. H. Yoshino, F. Zamponi, Phys. Rev. E 90, 022302 (2014)

20. P. Urbani, F. Zamponi, Phys. Rev. Lett. 118, 038001 (2017)

21. I. Goldhirsch, C. Goldenberg, Eur. Phys. Jour. E 9, 245 (2002)

22. J.P. Hansen, I.R. McDonald, Theory of Simple Liquids, 2nd edn. (Academic Press, London, 1986)

23. A. Nicolas, M. Fuchs, J. Non-Newt. Fluid Mech. 228, 64 (2016)

24. D.J. Evans, G. Morriss, Statistical Mechanics of Nonequilibrium Liquids (Cambridge University Press, London, 2008)

25. H. Risken, The Fokker-Planck Equation, 2nd edn. (Springer-Verlag, Berlin, 1989)

26. M. Priya, Th. Voigtmann, J. Rheol. 58, 1163 (2014)

27. C.P. Amann, M. Fuchs, J. Rheol. 58, 1191 (2014)

28. C.P. Amann, M. Siebenbürger, M. Ballauff, M. Fuchs, J. Phys.: Condens. Matter 27, $194121(2015)$

29. C.P. Amann, D. Denisov, M.T. Dang, B. Struth, P. Schall, M. Fuchs, J. Chem. Phys. 143, 034505 (2015)

30. N. Koumakis, M. Laurati, A.R. Jacob, K.J. Mutch, A. Abdellali, A.B. Schofield, S.U. Egelhaaf, J.F. Brady, G. Petekidis, J. Rheol. 60, 603 (2016)

31. A.R. Jacob, A.S. Poulos, S. Kim, J. Vermant, G. Petekidis, Phys. Rev. Lett. 115, $218301(2015)$

32. M. Siebenbürger, M. Ballauff, Th. Voigtmann, Phys. Rev. Lett. 108, 255701 (2012)

33. F. Frahsa, Ph.D. thesis, University of Konstanz 2015, http://nbn-resolving.de/ urn:nbn:de:bsz: $x x$

34. F. Frahsa, A.K. Bhattacharjee, J. Horbach, M. Fuchs, T. Voigtmann, J. Chem.Phys 138, 12A513 (2013)

35. M. Wang, J.F. Brady, Phys. Rev. Lett. 115, 158301 (2015)

36. T. Sentjabrskaja, P. Chaudhuri, M. Hermes, W. Poon, J. Horbach, S. Egelhaaf, M. Laurati, Sci. Rep. 5, 11884 (2015)

37. P. Chaudhuri, J. Horbach, Phys. Rev. E 88, 040301 (2013)

38. G.P. Shrivastav, P. Chaudhuri, J. Horbach, J. Rheol. 60, 835 (2016)

39. H. Jin, K. Kang, K.H. Ahn, J.K.G. Dhont, Soft Matter 10, 9470 (2014)

40. S. Mandal, M. Gross, D. Raabe, F. Varnik, Phys. Rev. Lett. 108, 098301 (2012)

41. A.M. Puertas, Th. Voigtmann, J. Phys.: Condens. Matter 26, 243101 (2014)

42. H. Wagner, D. Bedorf, S. Küchemann, M. Schwabe, B. Zhang, W. Arnold, K. Samwer, Nat. Mat. 10, 439 (2011)

43. A. Heuer, C.F.E. Schroer, D. Diddens, C. Rehwald, M. Blank-Burian, Eur. Phys. J. Special Topics 226, 3061 (2017)

44. M. Sperl, A. Zippelius, Eur. Phys. J. Special Topics 226, 3079 (2017)

45. S. Mandal, M. Spanner-Denzer, S. Leitmann, T. Franosch, Eur. Phys. J. Special Topics 226, 3129 (2017)

46. I. Gazuz, A.M. Puertas, Th. Voigtmann, M. Fuchs, Phys. Rev. Lett. 102, 248302 (2009) 
47. I. Gazuz, M. Fuchs, Phys. Rev. E 87, 032304 (2013)

48. C. Reichhardt, C.J.O. Reichhardt, Rep. Prog. Phys. 80, 026501 (2017)

49. S. Leitmann, T. Franosch, Phys. Rev. Lett. 118, 018001 (2017)

50. M. Gruber, G. Abade, A. Puertas, M. Fuchs, Phys. Rev. E 94, 042602 (2016)

51. T. Theenhaus, R. Schilling, A. Latz, M. Letz, Phys. Rev. E 64, 051505 (2001)

52. S. Lang, V. Boţan, M. Oettel, D. Hajnal, T. Franosch, R. Schilling, Phys. Rev. Lett. 105, $125701(2010)$

53. S.K. Schnyder, F. Höfling, T. Franosch, Th. Voigtmann, J. Phys.: Condens. Matter 23, $234121(2011)$

54. H. Yoshino, M. Mézard, Phys. Rev. Lett. 105, 015504 (2010)

55. A. Lemaître, Phys. Rev. Lett. 113, 245702 (2014)

56. J.P. Wittmer, H. Xu, J. Baschnagel, Phys. Rev. E 91, 022107 (2015)

57. S. Saw, P. Harrowell, Phys. Rev. Lett. 116, 137801 (2016)

58. E. Flenner, G. Szamel, Phys. Rev. Lett. 114, 025501 (2015)

59. R. Ahluwalia, S. Das, Phys. Rev. E 57, 5771 (1998)

60. D.H. Torchinsky, J.A. Johnson, K.A. Nelson, J. Chem. Phys. 136, 174509 (2012)

61. J. Chattoraj, A. Lemaître, Phys. Rev. Lett. 111, 066001 (2013)

62. J.C. Dyre, Rev. Mod. Phys. 78, 953 (2006)

63. B. Illing, S. Fritschi, D. Hajnal, C.L. Klix, P. Keim, M. Fuchs, Phys. Rev. Lett. 117, $208002(2016)$

64. A. Furukawa, H. Tanaka, Phys. Rev. E 84, 061503 (2011)

65. R.M. Puscasu, B.D. Todd, P.J. Daivis, J.S. Hansen, J. Chem. Phys. 133, 144907 (2010)

66. H.L. Peng, Th. Voigtmann, Phys. Rev. E 94, 042612 (2016)

67. S. Chowdhury, S. Abraham, T. Hudson, P. Harrowell, J. Chem. Phys. 144, 124508 (2016)

68. M. Maier, A. Zippelius, M. Fuchs, Phys. Rev., submitted

69. A. Nicolas, J. Rottler, J.L. Barrat, Eur. Phys. J. E 37, 50 (2014)

70. E.E. Ferrero, K. Martens, J.L. Barrat, Phys. Rev. Lett. 113, 248301 (2014)

71. C. Crauste-Thibierge, C. Brun, F. Ladieu, D. L'Hôte, G. Biroli, J.P. Bouchaud, Phys. Rev. Lett. 104, 165703 (2010)

72. T. Bauer, P. Lunkenheimer, A. Loidl, Phys. Rev. Lett. 111, 225702 (2013)

73. S. Albert, T. Bauer, M. Michl, G. Biroli, J.P. Bouchaud, A. Loidl, P. Lunkenheimer, R. Tourbot, C. Wiertel-Gasquet, F. Ladieu, Science 352, 1308 (2016)

74. R. Seyboldt, D. Merger, F. Coupette, M. Siebenbürger, M. Ballauf, M. Wilhelm, M. Fuchs, Soft Matter 12, 8825 (2016)

75. D. L'Hôte, R. Tourbot, F. Ladieu, P. Gadige, Phys. Rev. B 90, 104202 (2014)

76. J.G. Oldroyd, Proc. R. Soc. London, Ser. A 200, 523 (1950)

77. C. Derec, A. Ajdari, F. Lequeux, Eur. Phys. J. E 4, 355 (2001)

78. P. Coussot, Q.D. Nguyen, H.T. Huynh, D. Bonn, Phys. Rev. Lett. 88, 175501 (2002)

79. V.B. Nguyen, Phys. Rev. Lett. 107, 138303 (2011)

80. V. Mansard, A. Colin, P. Chaudhuri, L. Bocquet, Soft Matter 9, 7849 (2013)

81. H. Bodiguel, J. Beaumont, A. Machado, L. Martinie, H. Kellay, A. Colin, Phys. Rev. Lett. 114, $028302(2015)$

82. H. Cárdenas, Continuum Mechanics Simulations of the Nonlinear Deformation of Glass-Forming Fluids, $\mathrm{PhD}$ thesis, University of Düsseldorf, 2017, in preparation

83. M. Fuchs, M.E. Cates, Faraday Discuss. 123, 267 (2003)

84. Th. Voigtmann, Eur. Phys. J. E 34, 106 (2011)

85. S. Muller, R. Larson, E. Shaqfeh, Rheologica Acta 28, 499 (1989)

86. L. Casanellas, M.A. Alves, R.J. Poole, S. Lerouge, A. Lindner, Soft Matter 12, 6167 (2016)

87. S. Succi, The Lattice Boltzmann Equation for Fluid Dynamics and Beyond (Oxford University Press, Oxford, 2001)

88. B. Dünweg, A.J.C. Ladd, Adv. Polym. Sci. 221, 89 (2009)

89. S. Papenkort, Th. Voigtmann, J. Chem. Phys. 140, 164507 (2014)

90. S. Papenkort, Th. Voigtmann, J. Chem. Phys. 143, 044512 (2015) 
91. S. Papenkort, Th. Voigtmann, J. Chem. Phys. 143, 204502 (2015)

92. E.A.J.F. Peters, M.A. Hulsen, B.H.A.A. van den Brule, J. Non-Newt. Fluid Mech. 89, $209(2000)$

93. M.A. Hulsen, E.A.J.F. Peters, B.H.A.A. van den Brule, J. Non-Newt. Fluid Mech. 98, $201(2001)$

94. M. Tomé, M. de Araujo, M. Alves, F. Pinho, J. Comput. Phys. 227, 4207 (2008)

95. A. Nicolas, J.L. Barrat, Phys. Rev. Lett. 110, 138304 (2013)

96. P. Scheidler, W. Kob, K. Binder, J. Phys. Chem. B 108, 6673 (2004)

97. C.R. Nugent, K.V. Edmond, H.N. Patel, E.R. Weeks, Phys. Rev. Lett. 99, 025702 (2007)

98. H.B. Eral, D. van den Ende, F. Mugele, M.H.G. Duits, Phys. Rev. E 80, 061403 (2009)

99. M. Ozawa, W. Kob, A. Ikeda, K. Miyazaki, Proc. Natl. Acad. Sci. USA 112, 6914 (2015)

100. V. Krakoviack, Phys. Rev. E 84, 050501 (2011)

101. P. Jop, V. Mansard, P. Chaudhuri, L. Bocquet, A. Colin, Phys. Rev. Lett. 108, 148301 (2012)

102. F. Varnik, D. Raabe, Phys. Rev. E 77, 011504 (2008)

103. M. Frank, D. Anderson, E.R. Weeks, J.F. Morris, J. Fluid Mech. 493, 363 (2003)

104. S. Ghosh, F. Mugele, M.H.G. Duits, Phys. Rev. E 91, 052305 (2015)

105. S. Fritschi, Event-driven Brownian Dynamics Simulations of Two-dimensional Fluids far from Equilibrium, PhD thesis, University of Konstanz, 2017, in preparation.

106. A. Scala, Th. Voigtmann, C. De Michele, J. Chem. Phys. 126, 134109 (2007)

107. F. Weysser, D. Hajnal, Phys. Rev. E 83, 041503 (2011)

108. B. Illing, S. Fritschi, H. Kaiser, C.L. Klix, G. Maret, P. Keim, Proc. Natl. Acad. Sci. USA 114, $1856(2017)$

109. O. Henrich, F. Weysser, M.E. Cates, M. Fuchs, Phil. Trans. R. Soc. A 367, 5033 (2009)

110. J. Goyon, A. Colin, G. Ovarlez, A. Ajdari, L. Bocquet, Nature (London) 454, 84 (2008)

111. J. Goyon, A. Colin, L. Bocquet, Soft Matter 6, 2668 (2010)

112. S. Fritschi, M. Fuchs, Th. Voigtmann, Soft Matter 10, 4822 (2014)

113. M. Ballauff, J.M. Brader, S.U. Egelhaaf, M. Fuchs, J. Horbach, N. Koumakis, M. Krüger, M. Laurati, K.J. Mutch, G. Petekidis et al., Phys. Rev. Lett. 110, 215701 (2013)

114. D. El Masri, L. Berthier, L. Cipelletti, Phys. Rev. E 82, 031503 (2010)

115. K. Vollmayr-Lee, J.A. Roman, J. Horbach, Phys. Rev. E 81, 061203 (2010)

116. A.M. Puertas, M. Fuchs, M.E. Cates, Phys. Rev. E 75, 031401 (2007) 